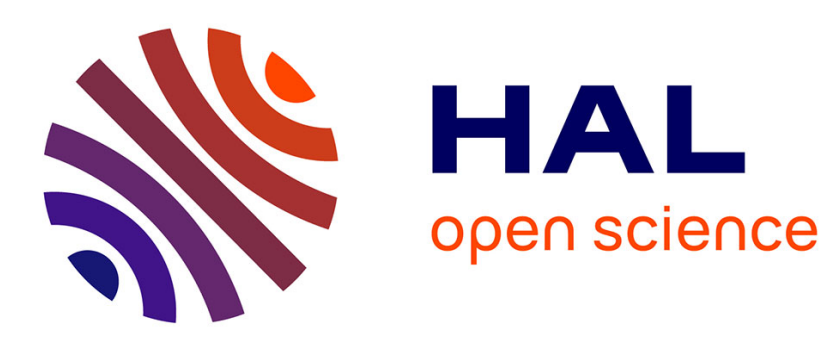

\title{
The glass and jamming transitions of soft polyelectrolyte microgel suspensions
}

\author{
Charlotte Pellet, Michel Cloitre
}

\section{To cite this version:}

Charlotte Pellet, Michel Cloitre. The glass and jamming transitions of soft polyelectrolyte microgel suspensions. Soft Matter, 2016, 12 (16), pp.3710-3720. 10.1039/c5sm03001c . hal-02404799

\author{
HAL Id: hal-02404799 \\ https://hal.science/hal-02404799
}

Submitted on 27 May 2021

HAL is a multi-disciplinary open access archive for the deposit and dissemination of scientific research documents, whether they are published or not. The documents may come from teaching and research institutions in France or abroad, or from public or private research centers.
L'archive ouverte pluridisciplinaire HAL, est destinée au dépôt et à la diffusion de documents scientifiques de niveau recherche, publiés ou non, émanant des établissements d'enseignement et de recherche français ou étrangers, des laboratoires publics ou privés. 


\title{
The glass and jamming transitions of soft polyelectrolyte microgel suspensions
}

\author{
Charlotte Pellet and Michel Cloitre
}

\begin{abstract}
We explore the influence of particle softness on the state diagram of well characterized polylectrolyte microgel suspensions using dynamic light scattering and rheology. Upon increasing the polymer concentration, we cross successively the well defined glass and jamming transitions which delimit four different states: dilute colloidal suspension, entropic glass, jammed glass, and dense glass. Each state has a specific dynamical fingerprint dictated by two key ingredients related to particle softness: elastic contact interactions, and osmotic or steric deswelling. Soft interactions control yielding and flow of the jammed glasses. The shrinkage of the microgels makes the glass transition look smoother than in hard sphere suspensions. We quantify the relation between the polymer concentration and the volume fraction, and show that the glass transition behaviour of soft microgels can be mapped onto that of hard sphere glasses once the volume fraction is used as the control parameter.
\end{abstract}

\section{Introduction}

The phase behaviour of colloidal suspensions becomes increasingly complex at large volume fractions. Fairly monodisperse Brownian hard sphere suspensions have long been considered as the archetypal problem in the field and have attracted a lot of interest. Colloidal hard spheres are rigid particles of submicron size that interact through the repulsive excluded volume potential only. They form glasses when their volume fraction exceeds a value of about $0.58 .^{1,2}$ In a glass, each particle can be seen as kinetically trapped in a cage formed its neighbours. Cages restrict macroscopic motion over a period of time exceeding the experimental time window over which the suspensions are observed. ${ }^{3}$ At the macroscopic scale, hard sphere glasses thus exhibit solid-like properties, i.e. reversible elastic deformation and resistance to flow at rest or at small applied stresses, but yield and flow at stresses exceeding the socalled yield stress. ${ }^{4}$ The magnitude of the elastic modulus is set by the thermal energy per particle, $k T / R^{3}$ where $R$ is the particle radius, resulting in very weak moduli of the order of a few pascals. The rheology of hard sphere glasses has motivated a lot of experimental studies. ${ }^{4-10}$ On the theoretical side, mode coupling theory (MCT) provides a microscopic description of the linear and non linear rheology of hard sphere suspensions and glasses. $^{11-14}$

Besides hard spheres, soft and deformable particles constitute an important class of colloids that are ubiquitous in practical applications: ${ }^{15}$ emulsions, microgels, multilamellar

Laboratoire Matière Molle et Chimie, (UMR 7167, ESPCI-CNRS) ESPCI ParisTech, 10 rue Vauquelin, 75005 Paris, France. E-mail: michel.cloitre@espci.fr vesicles, copolymer micelles, and star-like polymers. For Brownian particles, it is relevant to characterize the relative importance of elastic and thermal forces by the non dimensional parameter $\varepsilon=W / k T,{ }^{16-18}$ where $W$ is the elastic energy of the particles. Hard spheres are recovered in the limit $\varepsilon=\infty$ while ultrasoft particles such as star polymers correspond to $\varepsilon \approx 1$. At high density, soft particle suspensions exhibit non ergodicity and caged dynamics like hard spheres. ${ }^{19}$ However, while the volume fraction of fully random hard-sphere glasses cannot exceed close-packing at $\phi_{\mathrm{J}} \approx 0.64$, also termed the jamming point, ${ }^{16-21}$ soft particles can be packed at much higher volume fractions due to their deformability. In this regime, they experience soft interactions which are characterized by repulsive pair potentials of various forms. ${ }^{22-24}$ Soft particles can also undergo changes in volume in response to external stimuli like $\mathrm{pH}$ and temperature, or upon a concentration increase, because of osmotic or steric phenomena. ${ }^{25,26}$ Interdigitation is another important manifestation of softness that occurs when dangling chains are present at the periphery of the particles. ${ }^{27}$ Open and important questions are whether and how the interplay between softness and Brownian effects affects the phase behaviour and rheology of soft colloidal suspensions.

Today there is growing evidence that soft particle suspensions do not obey a universal description. Different scenarios have been reported. Emulsions can be considered as the simplest situation of soft suspensions. Emulsion droplets are quite rigid $\left(\varepsilon \approx 10^{6}-10^{7}\right)$ and deform at constant volume. They exhibit a glass transition at $\phi_{\mathrm{G}} \cong 0.58$, which is well described by $\mathrm{MCT}$, and a jamming transition around $\phi_{\mathrm{J}} \cong 0.64 .{ }^{28-31}$ Star polymer solutions are ultrasoft particles which involve all the components of softness, i.e ultrasoft interaction potential $\left(\varepsilon \approx 10^{1}-10^{2}\right)$, deformability, deswelling, and interdigitation. ${ }^{27}$ 
They exhibit a liquid to solid transition with the hallmarks of a glass transition, at a well defined concentration, without clear evidence for jamming. ${ }^{32}$ These experimental observations are qualitatively supported by recent simulations. ${ }^{17,33}$

Microgel particles constitute a broad class of colloids with intermediate softness $\left(\varepsilon \approx 10^{4}-10^{5}\right)$, which are tunable at will by varying the crosslinking density and the physicochemical environment. ${ }^{34}$ Aqueous suspensions of thermosensitive Nisopropylacrylamide (PNIPAm) core-shell particles have been found to be exquisite model systems for studying the flow behaviour of colloidal glasses with quantitative comparison to MCT theories. ${ }^{35-38}$ Different studies have interpreted the solidliquid transition of concentrated PNIPAm microgel suspensions as a thermal glass transition, ${ }^{39}$ an athermal jamming transition, ${ }^{40}$ or a combination of both. ${ }^{41}$ Simulations suggest that soft PNIPAm microgels should undergo a colloidal glass transition far from the jamming limit. ${ }^{33}$ Polyelectrolyte microgels form another important class of soft particles. Our former work in the field was mainly focused on concentrated suspensions where the rheology was described in the framework of an athermal micromechanical model. ${ }^{16}$ Experiments from other groups on crystallising polyelectrolyte microgels have shown that dynamical arrest is approached very gradually ${ }^{42}$ or suppressed, ${ }^{43}$ and that colloidal softness affects the phase diagram. ${ }^{44}$ It is thus evident that thorough investigations are highly desirable and timely, particularly with the aim of building a generic framework to rationalize the state diagram and the rheology of soft microgel suspensions.

In this work, we take-up on this challenge and report on the dynamics of well-characterized colloidal microgel suspensions, which encompass several key ingredients associated with softness: elasticity, deformability, and osmotic or steric deswelling. We analyze the state diagram and the rheological properties of the suspensions from very dilute to highly concentrated regime using light scattering, steady and oscillatory shear rheology at small and large amplitudes. In Section 2, we describe the microgel suspensions, their preparation and the experimental techniques and protocols which have been implemented. In Section 3 we present and discuss the experimental results. We identify the glass and jamming transitions which delimit dilute colloidal suspensions, entropic glasses and jammed glasses in the state diagram. A careful analysis of the rheological properties, combined with a simple model of swelling, provides a relation between the actual volume fraction and the microgel concentration. In Section 4, we conclude and highlight the impact of softness on the state diagram and suspension properties.

\section{Materials and methods}

\subsection{Microgel synthesis and characterization}

The suspensions used in this study were prepared from polyelectrolyte microgels in water. The microgels are spherical crosslinked polymer network synthesized by emulsion polymerisation at low $\mathrm{pH}(\cong 2)$ using ethyl acrylate (64 wt\%) and methacrylic acid (35 wt\%), and the difunctional crosslinker dicyclopentenyl-oxyethyl methacrylate. ${ }^{45}$ We implemented a semi-batch technique in starved-feed conditions and continuous feeding of initiator and reactants. The conditions of synthesis (temperature, feeding rate) were optimized to yield constant conversion rate and linear particle growth with time. These conditions are known to reduce inhomogeneties of composition and limit the occurrence of dangling ends. ${ }^{46,47}$ In the following we will thus consider that our microgels are uniform in composition. This is in contrast with other systems studied in the literature which consist of a soft brush-like shell coupled to a dense core. ${ }^{48}$ The average number of monomeric units between two crosslinks is of the order of 128 assuming a uniform distribution of crosslinks. Unreacted monomers, surfactants and other impurities present in the product of the synthesis were removed by ultrafiltration. The solid content of the stock suspension was determined by thermogravimetry. Microgels suspensions were prepared by diluting a volume of stock solution at the desired concentration and adding sodium hydroxide which ionizes the methacrylic acid units and provokes the swelling of the particles. The degree of neutralization is characterized by the molar ratio $f$ between $\mathrm{NaOH}$ and $\mathrm{COOH}$ functions; $f=1$ in all the experiments reported below.

The hydrodynamic radius, the radius of gyration and the size distribution of the microgels were determined by light scattering (Section 2.3). At low $\mathrm{pH}$ where the microgels are collapsed, the radius of gyration determined using a Guinier plot of the static intensity in the range $q R_{\mathrm{g}}<1$ is $R_{\mathrm{g}} \cong 60 \mathrm{~nm}$; the hydrodynamic radius measured by dynamic light scattering is $R_{\mathrm{h}} \cong 78 \mathrm{~nm}$. The particles thus behave very much like hard spheres $\left(R_{\mathrm{g}} / R_{\mathrm{h}} \cong 0.8\right)$. The particle distribution is weakly polydisperse with a mean-square deviation of about 0.1 . For $f=1$, swollen microgels have a hydrodynamic radius $R_{\mathrm{h}} \cong 305$ $\mathrm{nm}$; the swelling ratio is $Q \cong 60$. In the limit $C \rightarrow 0$, the polymer concentration $C$ and the particle volume fraction $\phi$ are proportional: $\phi_{0}=k C$ with $k=0.11 \pm 0.01 \mathrm{~g} / \mathrm{mg}$ (Section 3.6). The coefficient $k$ can be expressed as: $k=m / v \rho_{S}$ where $m$ is the mass of a microgel, $v$ its volume $\left(=4 \pi R_{\mathrm{h}}^{3} / 3\right)$, and $\rho_{S}$ the suspension density which is equal to the water density. We deduce easily the mass of a microgel $\left(m=1.2 \times 10^{-15} \mathrm{~g}\right)$, the total polymer volume fraction and the methacrylic acid concentration in the collapsed and swollen states $\left(\varphi_{0}=0.7\right.$ and $\varphi \cong 0.01 ; c_{0}=2 \mathrm{M}$ and $\mathrm{c}=0.03 \mathrm{M}$ ), in fairly good agreement with previous reports. ${ }^{25,45}$ At higher concentrations, microgels are able deswell osmotically so that the volume fraction is not known a priori. We will use the polymer concentration, $C$, as the control variable $(0.1 \mathrm{mg} / \mathrm{g}<C<50 \mathrm{mg} / \mathrm{g})$.

\subsection{Rheological measurements}

Rheological measurements were carried out using an Anton Paar MCR 501 rheometer. For the most dilute samples $(C<12$ $\mathrm{mg} / \mathrm{g}$ ) the rheometer was equipped with a double-wall Couette geometry (internal cylinder diameter: $23.820 \mathrm{~mm}$; external cylinder diameter: $27.594 \mathrm{~mm}$; capacity: $\left.3.9 \mathrm{~cm}^{3}\right)$. For concentrated samples $(C \geq 12 \mathrm{mg} / \mathrm{g})$ the rheometer was mounted with a cone and Peltier plate geometry with a diameter 
of $50 \mathrm{~mm}$, a $2^{\circ}$ angle, and a truncation of $48 \mu \mathrm{m}$. The shearing surfaces were sandblasted to provide a surface roughness of 2 to $4 \mu \mathrm{m}$, which prevented the occurrence of slip. A solvent trap was placed around the sample to minimize water evaporation, and the interior atmosphere of the trap was saturated using a small volume of distilled water. All measurements were made at $20.0 \pm 0.1^{\circ} \mathrm{C}$. Prior to all measurements, suspensions were presheared at a shear rate of $500 \mathrm{~s}^{-1}$ for $30 \mathrm{~s}$ in order to erase the mechanical history and place them in a reproducible state. Before frequency sweep tests, which needed to be performed close to equilibrium conditions, they were kept at rest for a waiting time of about 4 hours to make aging effects negligible. ${ }^{49}$ We performed two types of rheological tests: steady shear experiments and oscillatory measurements.

Steady shear experiments, where the stress $\sigma$ was measured as a function of the applied shear rate $\dot{\gamma}$, were performed by applying constant shear rates varying from $\cong 10^{3}$ to $10^{-4} \mathrm{~s}^{-1}$ and recording the stress when steady state was reached. To quantitatively analyze flow curves $\sigma(\dot{\gamma})$, the experimental data were fitted to various phenomenological models. Dilute suspensions $(C<11 \mathrm{mg} / \mathrm{g})$ had a purely viscous behaviour; the nonlinear steady-shear data were modelled using a version of the Carreau-Yasuda viscosity model which included a term for the viscosity at infinite shear-rate and a single slow relaxation process: ${ }^{50}$

$$
\sigma(\dot{\gamma})=\dot{\gamma}\left[\eta_{\infty}+\left(\eta_{0}-\eta_{\infty}\right)\left(1+\left(\dot{\gamma} \tau_{0}\right)^{a}\right)^{b}\right]
$$

$\eta_{0}$ and $\eta_{\infty}$ are the low and high shear rate limits of the viscosity, respectively; $\tau_{0}$ is the longest relaxation time which marks the onset of shear thinning; $a$ and $b$ are two fitting parameters. The Carreau-Yasuda model was implemented by minimizing the residuals between Eq. (1) and the experimental stress data using ordinary least-squares fitting.

At high concentrations $(C \geq 16 \mathrm{mg} / \mathrm{g})$, the suspensions exhibited yielding properties and the flow curves were fitted to the Herschel-Bulkley equation: ${ }^{19}$

$$
\sigma(\dot{\gamma})=\sigma_{y}+k \dot{\gamma}^{n}
$$

where $\sigma_{\mathrm{y}}$ is the dynamic yield stress, $k$ the consistency parameter, and $n$ a real exponent. At intermediate concentrations $(11 \mathrm{mg} / \mathrm{g}<C<16 \mathrm{mg} / \mathrm{g})$, we used a form which involves a linear combination of a constant yield stress and two power law variations:

$$
\sigma(\dot{\gamma})=\sigma_{y}+k^{\prime} \dot{\gamma}^{p}+k \dot{\gamma}^{n}
$$

where $k, k, n$, and $p$ are parameters. Similar functional forms have been successfully used to describe the flow curves of glassy star polymers in good solvent conditions ${ }^{32}$ and hard sphere entropic glasses. ${ }^{37}$

Oscillatory frequency sweeps were used to measure the variations of the storage modulus $G^{\prime}$ and loss modulus $G^{\prime \prime}$ as functions of the angular frequency $\omega\left(10^{-2}<\omega<10^{2} \mathrm{rad} / \mathrm{s}\right)$ at small strain amplitudes in the linear viscoelastic regime $\left(\gamma_{0}=6 \times 10^{-3}\right)$. The storage and loss moduli show the characteristic variations exhibited by many soft materials, i.e. a nearly constant plateau in $G^{\prime}(\omega)$ and a much lower $G^{\prime \prime}(\omega)$ with a small minimum around a frequency $\omega_{\mathrm{m}}$. We defined the plateau modulus of the suspension, $G_{0}$, as the value of $G^{\prime}(\omega)$ at $\omega_{\mathrm{m}}$. Oscillatory strain sweep experiments were performed by applying a periodic strain at a fixed angular frequency $\omega=1 \mathrm{rad} / \mathrm{s}$ in order to obtain the variations of $G^{\prime}$ and $G^{\prime}$ ' with the strain amplitude $\gamma_{0}\left(10^{-3}<\gamma_{0}<10\right)$.

\subsection{Dynamic light scattering}

The microgel suspensions used in this study are visually transparent so that standard light scattering could be used to characterize their dynamical properties. The setup was a CGS3 Compact Goniometer System from ALV, equipped with a digital correlator and a Helium-Neon laser emitting at $632.8 \mathrm{~nm}$. After preparation the suspensions were transferred into cylindrical borosilicate test tubes (diameter: $10 \mathrm{~mm}$ ) commercially available from VWR. The sample tubes were inserted in a thermostated cuvette filled with toluene which was placed on a motor-driven precision goniometer $\left( \pm 0.01^{\circ}\right)$ enabling the photomultiplier detector to rotate accurately from $20^{\circ}$ to $150^{\circ}$ scattering angles $\left(1.4 \times 10^{-3} \mathrm{~nm}^{-1}<q<7.8 \times 10^{-2} \mathrm{~nm}^{-1}\right)$. The time-averaged autocorrelation function of the light intensity at a scattering vector $\boldsymbol{q}$ was computed using a digital correlator (ALV-LSE 5004): $g_{t}^{(2)}(\boldsymbol{q}, t)=\langle I(\boldsymbol{q}, t+\tau) I(\boldsymbol{q}, \tau)\rangle /$ $\langle I(\tau)\rangle^{2}$. For $C \leq 11 \mathrm{mg} / \mathrm{g}$, the suspensions were ergodic and the time averaged autocorrelation functions were equivalent to ensemble-averaged autocorrelation functions. The field autocorrelation function $g_{t}^{(1)}(\boldsymbol{q}, t)=\langle E(\boldsymbol{q}, t+\tau) E(\boldsymbol{q}, \tau)\rangle /$ $\langle E(\tau)\rangle^{2}$ is related to the intensity autocorrelation function through the Siegert relation: $g_{t}^{(2)}(\boldsymbol{q}, t)=1+\beta\left[g_{t}^{(1)}(\boldsymbol{q}, t)\right]^{2}$. The coherence factor of our set up was measured using dilute polystyrene sphere standards: $\beta=0.90 \pm 0.02$. This value is unchanged up to the largest microgel concentrations investigated, indicating that multiple light scattering does not significantly affect our measurements. The measurement time was of $300 \mathrm{~s}$ at low concentrations and was increased to $2000 \mathrm{~s}$ near the non ergodic transition. When the suspensions were non-ergodic, the time average correlation functions did not give directly access to the ensemble-averaged correlation functions. We used the Pusey-Schätzel method which relates the ensemble averaged field autocorrelation function to time-averaged correlation functions: ${ }^{51}$

$$
g_{e}^{(1)}(\boldsymbol{q}, t)=1+\frac{\langle I\rangle_{t}}{\langle I\rangle_{e}}\left(\sqrt{\frac{g_{t}^{(2)}(\boldsymbol{q}, t)-g_{t}^{(2)}(\boldsymbol{q}, 0)}{\beta}+1}-1\right)
$$

$\langle I\rangle_{\mathrm{t}}$ is the time-averaged intensity associated with a single measurement of $g_{t}^{(2)}(\boldsymbol{q}, t)$ at a fixed wavevector $\boldsymbol{q}$ and $\left\langle I>_{\mathrm{e}}\right.$ is the ensemble-averaged intensity obtained by rotating the cylindrical tube containing the sample around its axis (duration: $300 \mathrm{~s}$; angular velocity: $1 \mathrm{rpm}$ ).

The correlation functions were analyzed via inverse Laplace transformation which, assuming a superposition of exponentials, determines a continuous spectrum of relaxation times. The characteristic relaxation times associated with different relaxation modes correspond to the maximum values of the spectrum. The inverse Laplace transform was computed using 
CONTIN allowing for the determination of the relaxation times and intensity of the modes associated with the relaxation spectrum. $^{52}$

\section{Results and discussion}

\subsection{Local dynamics probed by Dynamic Light Scattering}

Figure 1 shows the ensemble-averaged field autocorrelation functions $g_{e}^{(1)}(t)$ measured at $\theta=90^{\circ}$ over a wide range of samples spanning two decades in concentration. At low concentrations, the field autocorrelation functions are well described by single exponentials. The relaxation rates are proportional to $q^{2}$ (data not presented), indicating simple diffusive behaviour with the self-diffusion coefficient $D$ independent of the wavevector q. $D$ decreases when the concentration increases (Fig. 1). Extrapolation to zero concentration yields the single particle self-diffusion coefficient: $D_{0}=7.1 \times 10^{-13} \mathrm{~cm}^{2} / \mathrm{s}$. From the Stokes-Einstein-Sutherland relation $D_{0}=k T / 6 \pi \eta_{\mathrm{S}} R\left(\eta_{\mathrm{S}}=0.98 \mathrm{mPa} . \mathrm{s}\right.$ is the water viscosity at $20^{\circ} \mathrm{C}$ ), we deduce the microgel radius at infinite dilution: $R=305 \pm 10 \mathrm{~nm}$. Above $C=8 \mathrm{mg} / \mathrm{g}$, the shape of $g_{e}^{(1)}(t)$ changes and a second decay appears at long time. The diffusion coefficients depend on the wavevector due to the presence of interparticle correlations. They decrease with increasing $q$, go through a weak minimum, before reaching constant values at large $q R$ values. This behaviour is qualitatively similar to that found in other colloidal suspensions. ${ }^{53-56} \mathrm{We}$ can assign the fast and slow modes when $q R>6$ to the short and long time self diffusion, respectively. The short and long time self-diffusion coefficients, $D_{\mathrm{S}}$ and $D_{\mathrm{L}}$, are represented in the inset of Fig. 1. When the concentration is larger than $C=11 \mathrm{mg} / \mathrm{g}$, the autocorrelation functions no longer decay to zero over the experimental time window and exhibit a frozen-in component which rapidly levels off with concentration. This behaviour is the one expected for a system undergoing a colloidal glass transition. The onset of non ergodicity will be designated by $C_{\mathrm{G}}$ in the following and we will take $C_{\mathrm{G}} \cong 11 \mathrm{mg} / \mathrm{g}$.

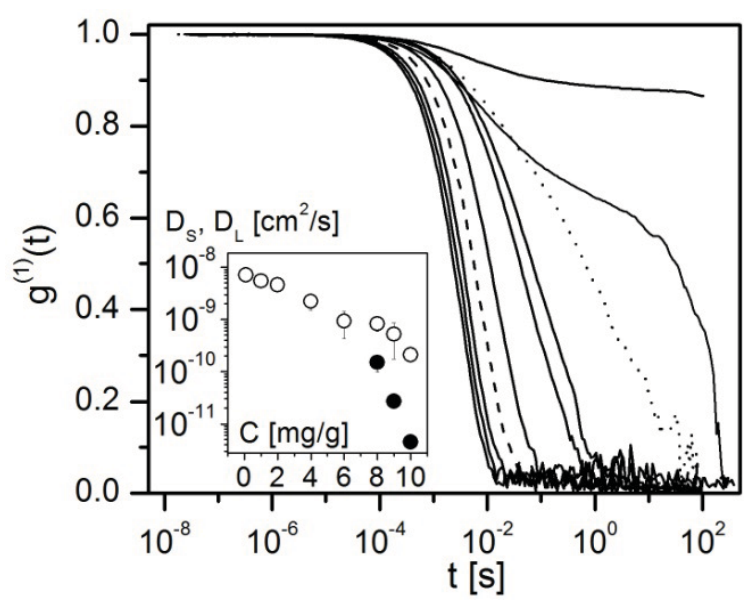

Fig. 1 Ensemble-averaged field autocorrelation functions for suspensions with $C=12,11,10$ (...), 9, 8, 6, $4(---), 2,1,0.1 \mathrm{mg} / \mathrm{g}$ (from right to left) at $q=0.021$ $\mathrm{nm}^{-1}\left(q R_{\mathrm{h}} \cong 6\right)$. The inset shows the variations of the short $(O)$ and long ( time self-diffusion coefficients, $D_{\mathrm{S}}$ and $D_{\mathrm{L}}$, measured for $q R_{\mathrm{h}}>6$.



3.2 Small amplitude oscillatory shear rheology (SAOS)

To corroborate the nature of the non-ergodic transition occurring at $\mathrm{C}_{\mathrm{G}}$, we measured the frequency dependence of the loss and storage moduli, $G^{\prime}$ and $G^{\prime \prime}$, for six increasing concentrations. The results are plotted in Fig. 2. For the $8 \mathrm{mg} / \mathrm{g}$ sample there is a terminal regime which extends over almost the entire frequency window, indicating that the sample is a viscous solution; $G$ ' and $G$ ' nearly coincide at high frequencies. For $C=10.5 \mathrm{mg} / \mathrm{g}$, the dispersion is a viscoelastic liquid with the onset of terminal behaviour detected at very low angular frequency $\omega=10^{-2} \mathrm{rad} / \mathrm{s}$ and a weak elastic plateau at higher frequency. The storage moduli for $C=11 \mathrm{mg} / \mathrm{g}$ and $C=12$ $\mathrm{mg} / \mathrm{g}$ are larger than the respective loss moduli over the entire frequency window revealing weak solid-like properties. For these two concentrations, the plateau modulus is not perfectly constant but increases with the angular frequency. Moreover the onset of terminal relaxation remains visible at the lowest frequency investigated. For $C \geq 14 \mathrm{mg} / \mathrm{g}$, the plateau modulus is constant over the entire frequency window and terminal behaviour is shifted to much lower frequencies which fall outside the frequency window probed by rheology.

Based on the frequency sweep data presented in Fig. 2, we determine the plateau shear modulus $G_{0}$ of the different samples as explained in Section 2.2. The variations of $G_{0}$ with the concentration are reported in Table 1 and plotted in Fig. 3. Near the onset of non ergodicity at $C_{\mathrm{G}}, G_{0}$ is of the order of the characteristic modulus $k T / R^{3}$ associated with entropic elasticity $(\sim 1 \mathrm{~Pa}) . G_{0}$ increases moderately up to a concentration $C_{\mathrm{J}}$ where it starts to rise sharply with a linear dependence on concentration. We estimate the crossover $C_{\mathrm{J}}=16 \mathrm{mg} / \mathrm{g}$ from the intercept of the line with the data at lower concentrations. For $C>40 \mathrm{mg} / \mathrm{g}$, the data start to deviate from linearity, suggesting a possible change of behaviour at large concentration. 


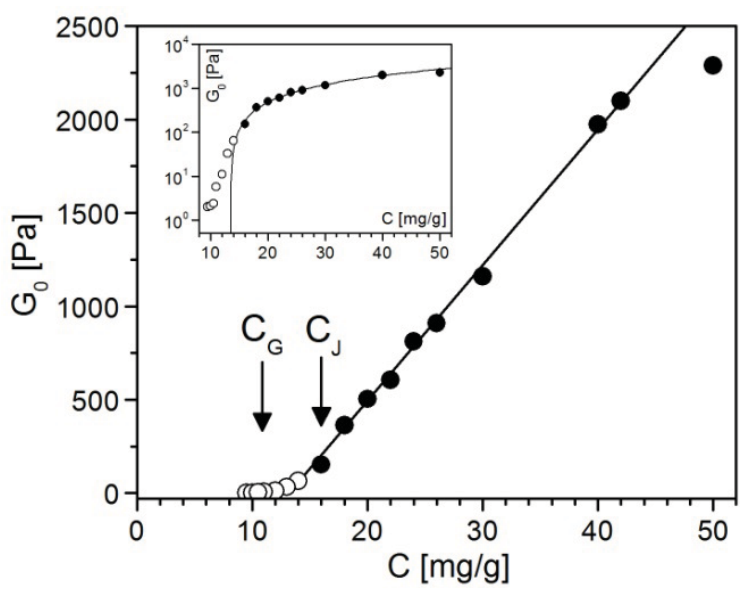

Fig. 3 Variations of the plateau modulus of microgel suspensions in the entropic glass (O) and jammed glass $(\mathbf{O})$ regimes versus the polymer concentration. The black arrows in the main graph point to the glass and jamming transitions. The inset shows the data in lin-log representation. The solid lines are the best fit of the data to a linear variation in the jammed glass region.

These results give evidence for distinct dynamical states. Below $C_{\mathrm{G}}$, dynamic light scattering experiments and linear rheology indicate that the samples behave like dilute colloidal suspensions. We associate the concentration $C_{\mathrm{G}}$ to the entropic glass transition and $C_{\mathrm{J}}$ to the jamming transition. The domain $C_{\mathrm{G}}<C<C_{\mathrm{J}}$ corresponds to the entropic glass regime. The particles are trapped in metastable cages which restrict longrange displacements on time scale larger than the experimental time window. Solid-like behaviour is due to the existence of elastic restoring forces of entropic origin which drive the particles back to their equilibrium position when they are sheared. Above $C_{\mathrm{J}}$, the microgel dispersions still have an amorphous structure but the particles adapt their shape to steric constraints and develop flat contacts through which they exert repulsive forces of elastic origin. ${ }^{45}$ In this regime microgel suspensions can be viewed as disordered packings of athermal frictionless particles. ${ }^{20}$ The moduli, which are controlled by elastic contact forces, increase rapidly with concentration because both the number of contacts per particle and the compression of particles increase. ${ }^{24,45}$ The linear variation observed in Fig. 3 agrees with predictions from simulations for athermal elastic spheres in the jammed regime. ${ }^{22,24}$

\subsection{Large amplitude oscillatory shear experiments (LAOS)}

We conducted systematic strain sweep experiments (LAOS). In Fig. 4, we represent the variations of the storage and loss moduli, $G^{\prime}$ and $G^{\prime \prime}$, and of the stress amplitude, $\sigma$, with the strain amplitude, $\gamma_{0}$, when $C_{\mathrm{G}}=11 \mathrm{mg} / \mathrm{g}$, i.e. at the onset of the entropic glass regime, and $\omega=1 \mathrm{rad} . \mathrm{s}^{-1}$. The results for the other concentrations and angular frequencies are qualitatively the same. G', G', and $\sigma$ have the characteristic shapes expected for yield stress materials like soft particle glasses, ${ }^{57}$ emulsions, ${ }^{58}$ star polymer solutions, ${ }^{32}$ and hard sphere glasses. ${ }^{9}$ At low strain amplitudes, the suspension exhibits solid-like behaviour with $G^{\prime}>G^{\prime}$; both moduli are independent of the strain amplitude and the stress varies linearly with the strain, indicating that the rheological response is in the linear regime. At larger amplitudes $G$ ' decreases whereas $G$ ' goes through a

\begin{tabular}{ccccccc}
\hline $\begin{array}{c}C \\
(\mathrm{mg} / \mathrm{g})\end{array}$ & $\begin{array}{c}G_{0} \\
(\mathrm{~Pa})\end{array}$ & $\mu$ & $v$ & $m$ & $\begin{array}{c}\sigma_{\mathrm{y}} \\
(\mathrm{Pa})\end{array}$ & $\begin{array}{c}\gamma_{y} \\
(-)\end{array}$ \\
\hline 11 & 5.7 & 1.21 & 0.54 & 0.43 & 0.28 & 0.049 \\
12 & 11 & 1.22 & 0.57 & 0.41 & 0.53 & 0.047 \\
13 & 32 & 1.28 & 0.63 & 0.28 & 2.49 & 0.040 \\
14 & 66 & 1.32 & 0.67 & 0.28 & 2.7 & 0.043 \\
16 & 153 & 1.36 & 0.75 & 0.23 & 9.5 & 0.061 \\
18 & 363 & 1.37 & 0.78 & 0.20 & 23 & 0.064 \\
20 & 504 & 1.38 & 0.76 & 0.17 & 35 & 0.069 \\
22 & 606 & 1.38 & 0.76 & 0.16 & 40 & 0.066 \\
24 & 812 & 1.38 & 0.76 & 0.16 & 64 & 0.079 \\
26 & 909 & 1.38 & 0.75 & 0.15 & 71 & 0.078 \\
30 & 1160 & 1.37 & 0.74 & 0.16 & 95 & 0.082 \\
40 & 1974 & 1.25 & 0.72 & 0.15 & 171 & 0.092 \\
50 & 2290 & 1.18 & 0.67 & 0.21 & 218 & 0.095
\end{tabular}

Table 1 Values of experimental parameters determined from SAOS and LAOS experiments in the entropic and jammed regimes $\left(\omega=1 \mathrm{rad} . \mathrm{s}^{-1}\right)$.

maximum before declining and crossing the storage modulus: the onset of non linearity is associated with yielding. At even larger amplitudes both moduli decrease as $G^{\prime}\left(\gamma_{0}\right) \propto \gamma_{0}^{-\mu}$ and $G^{\prime \prime}\left(\gamma_{0}\right) \propto \gamma_{0}^{-v}$. In the high strain limit, the stress is well fitted to a power-law $\sigma\left(\gamma_{0}\right) \propto \gamma_{0}^{-m}$ which is represented by a straight line in double logarithmic coordinates. The intersection between the low strain linear variation and the high strain power law variation marks the intersection between the liquidlike and the solid-like behaviours. This method provides a quantitative determination of the yield strain and stress which will be discussed in Section 3.5.
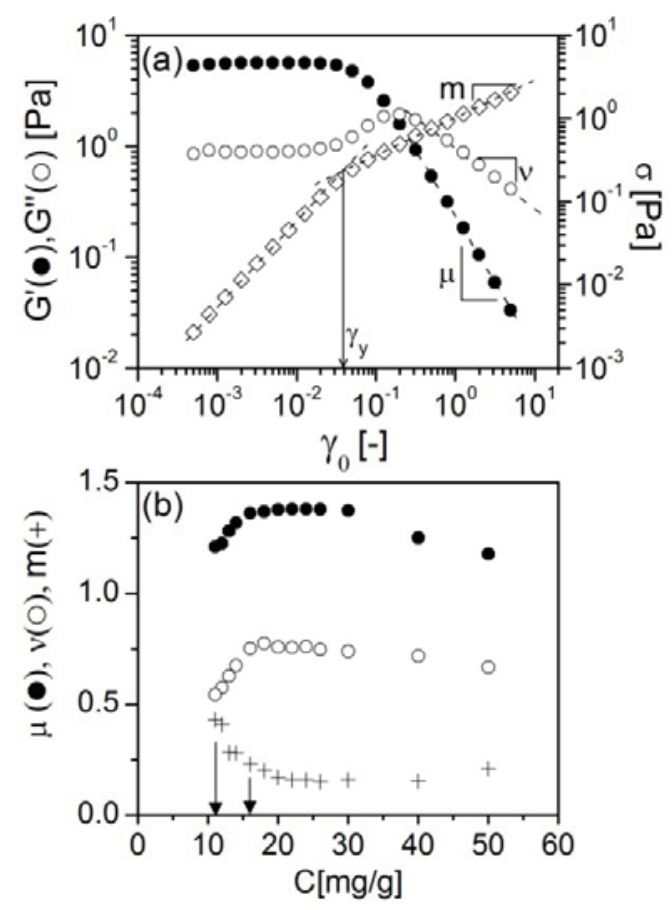

Fig. 4 (a) Dynamic strain sweep at $\omega=1 \mathrm{rad} / \mathrm{s}$ of the microgel suspension with $\mathrm{C}=11 \mathrm{mg} / \mathrm{g} ; \mu, v$, and $m$ are the exponents of the power law variations for the storage modulus $(\bullet)$, loss modulus $(O)$ and shear stress $(\square)$ at large strains. (b) Variations of $\mu(\bullet) \vee(O)$, and $m(+)$ versus polymer concentration; the arrows point to the entropic glass $\left(C_{G} \cong 11 \mathrm{mg} / \mathrm{g}\right.$ ) and jammed glass $\left(C_{J} \cong 16 \mathrm{mg} / \mathrm{g}\right.$ ) transitions as explained in the text. 
We performed systematic LAOS experiments for microgel concentrations in the entropic glass and jammed glass regimes. The variations of $\mu, v$, and $m$ are reported in Table 1 and plotted in Fig. 4b. Above $C_{\mathrm{G}}=11 \mathrm{mg} / \mathrm{g}$, both $\mu$ and $v$ first increases before reaching plateau values, $\mu=1.38 \pm 0.01$ and $v=0.76 \pm$ 0.01 , at $C_{\mathrm{J}}=16 \mathrm{mg} / \mathrm{g}$. At the largest concentrations investigated, we observe a slight decrease in $\mu$ and $v$. It is interesting to note that the ratio $\mu / v$ takes values close to $2(\mu / \nu=1.82 \pm 0.02$ in the jammed glass regime) as observed in many soft materials. ${ }^{32,57}$ The variations of the exponent $m$ also allow us to distinguish the entropic and jammed glass regimes. $m$ decreases steadily in the entropic glass regime before plateauing at a value of about 0.2 in the jammed glass regime. In conclusion, LAOS experiments provide an efficient way to identify thermal and jammed glasses as well as the associated transitions.

\subsection{Steady-shear rheology in the dilute suspension regime}

We used steady-shear rheology to characterize the flow behaviour of microgel suspensions and glasses over several decades in applied shear rates and a wide range of concentrations. Figure 5 depicts the variations of the steadystate shear stress $\sigma$ as a function of the applied shear rate $\dot{\gamma}$ in the dilute colloidal suspension regime when the concentration is smaller than the glass transition concentration $C_{\mathrm{G}}$. For $C<2$ $\mathrm{mg} / \mathrm{g}$ the suspensions exhibit Newtonian behaviour with the stress being proportional to the shear rate over the entire range of shear rates. At higher concentrations, Newtonian behaviour is still observed at low shear rates but the suspensions become shear-thinning at high shear rates. The onset of shear-thinning is shifted to low shear rates as the concentration increases. The flow curve for $C_{\mathrm{G}}=11 \mathrm{mg} / \mathrm{g}$ does not exhibit terminal behaviour over the experimental window and marks the limit of the dilute suspension regime. This is in agreement with the appearance of non-ergodicity in light scattering experiments (Fig. 1) and the emergence of solid-like response in the linear viscoelastic experiments (Fig. 2).

We determined the low-shear and high shear rates viscosities, $\eta_{0}$ and $\eta_{\infty}$, as well the longest characteristic time $\tau_{0}$ which marks the onset of terminal behaviour by fitting the

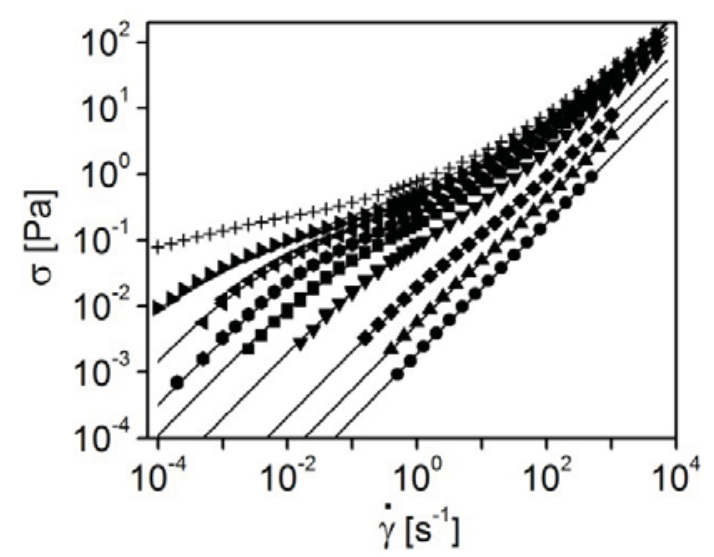

Fig. 5 Flow curves for microgel suspensions in the dilute suspension regime. The polymer concentration increases from bottom to top: $C=2(\bullet), 4(\boldsymbol{\Delta}), 6(\bullet), 8(\boldsymbol{\nabla}), 9$ $(\mathbf{\square}), 9.5(-), 10(4), 10.5(-)$, and $11(+) \mathrm{mg} / \mathrm{g}$. The black lines represent the fits of the experimental data to Eq (1). experimental data to the Carreau-Yasuda equation (1). Below $C=2 \mathrm{mg} / \mathrm{g}$, only $\eta_{0}$ can be determined whereas at larger concentrations, we are able to determine $\eta_{0}, \eta_{\infty}$ and $\tau_{0}$ up to $C=10.5 \mathrm{mg} / \mathrm{g}$. For $C=11 \mathrm{mg} / \mathrm{g}$, the terminal regime falls outside the accessible shear-rate window and $\eta_{0}$ is no longer determined. The variations of the relative zero-shear and high shear viscosities, $\eta_{0} / \eta_{\mathrm{S}}$ and $\eta_{\infty} / \eta_{\mathrm{S}}$, are plotted in Fig. 6 against the polymer concentration. $\eta_{0} / \eta_{\mathrm{S}}$ increases steadily by five orders of magnitude over the range of concentration where it can be determined. The inset is an enlargement of the very low concentration regime for $C<2 \mathrm{mg} / \mathrm{g}$ where the relative low shear viscosity is well described by a second order polynomial of the form $\eta_{0} / \eta_{\mathrm{S}}=\beta_{0}+\beta_{1} C+\beta_{2} C^{2}$ with $\beta_{0}=1 \pm 0.001$, $\beta_{1}=0.26 \pm 0.02$, and $\beta_{2}=1.1 \pm 0.3$. The relative high shear viscosity, $\eta_{\infty} / \eta_{\mathrm{S}}$, increases more slowly than the relative lowshear viscosity and lays well below it. The longest characteristic time, $\tau_{0}$, follows the variation of the low-shear viscosity within a constant prefactor.

To connect these macroscopic results to the local dynamics properties depicted in Fig. 1, we calculate the characteristic time that a microgel takes to diffuse in the environment formed by its neighbours over a distance comparable to its radius: $\tau_{0}^{\prime}=R^{2} / D_{L}$. Remarkably, $\tau_{0}$ and $\tau_{0}^{\prime}$ coincide as shown in Fig. 6 . This demonstrate that the onset of terminal regime is set by the structural time $\tau_{0}^{\prime}$. When $\tau_{0}^{\prime}$ is smaller than the hydrodynamic time $\dot{\gamma}^{-1}$, Brownian motion controls the local structure and the suspension is Newtonian. When $\tau_{0}^{\prime} \gg \dot{\gamma}^{-1}$, the applied flow modifies the equilibrium structure and the suspension is shearthinning. $D_{\mathrm{L}}$ is given by the generalized Stokes-EinsteinSutherland relation, ${ }^{27,54} D_{\mathrm{L}}=k T / 6 \pi \eta_{0} R$, which involves the suspension zero shear viscosity. It follows that $\tau_{0}^{\prime}=\tau_{0}$ and $\eta_{0} / \eta_{\mathrm{S}}$ are proportional through a coefficient of the order of 0.1 , just as found in Fig. 6.

To close this section, it is important to note that the behaviour shown in Fig. 6 is qualitatively similar to that of other soft particle suspensions like star polymers, ${ }^{32}$ and copolymer micelles. ${ }^{59}$ It marks a distinct feature of soft spheres in comparison to hard sphere suspensions. In hard spheres, the viscosity first increases slowly before diverging making it very

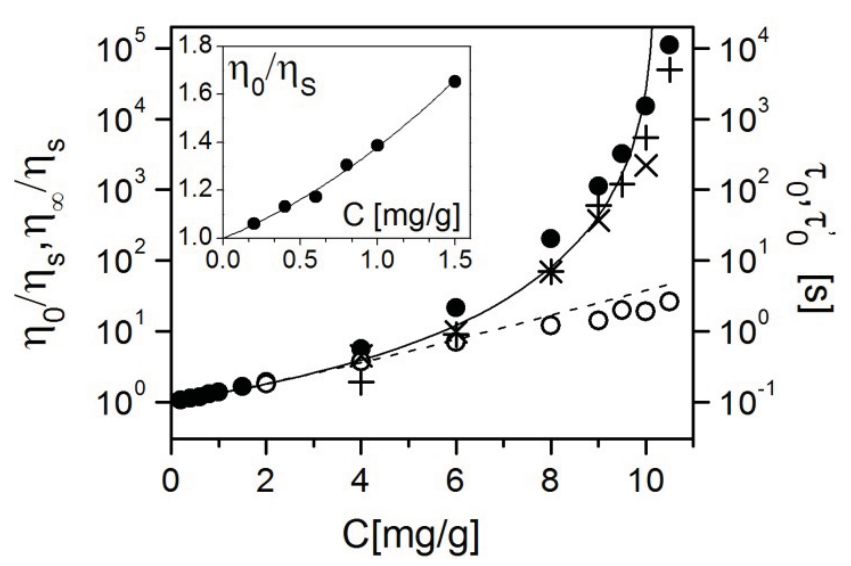

Fig. 6 Variations of the relative zero shear rate viscosity $\left(\eta_{0} / \eta_{s} ; \mathbf{0}\right)$, high shear rate viscosity $\left(\eta_{\infty} / \eta_{\mathrm{s}} ; \bigcirc\right)$, longest relaxation time $\left(\tau_{0} ;+\right)$, and structural time $\left(\tau_{0}^{\prime} ; \times\right)$. The continuous line represents Eq. A2 calculated using the $\phi(C)$ relation established in Section 3.6 with $\phi_{G}=0.615$. The dotted line represents Eq. A4. The inset is an enlargement showing the zero-shear viscosity in the low concentration limit. 
difficult to reach very high viscosities in a controlled way. In soft spheres the increase of the low shear viscosity is progressive and it is possible to achieve very high value of $\eta_{0} / \eta_{\mathrm{s}}$. This result will be interpreted below in Section 3.6.

\subsection{Flow and yielding through the thermal and jammed glass regimes}

Figure 7 shows the flow curves at high concentrations. They do not extrapolate to zero stress at low shear rate, supporting the existence of a dynamical yield stress $\sigma_{\mathrm{y}}$. Moreover, we observe a clear qualitative difference between the flow curves of samples just above $C_{\mathrm{G}}=11 \mathrm{mg} / \mathrm{g}$, i.e. in the entropic glass regimes, and those at higher concentrations than $C_{\mathrm{J}}=16 \mathrm{mg} / \mathrm{g}$ in the jammed glass regime.

In the entropic regime $\left(C_{\mathrm{G}}<C<C_{\mathrm{J}}\right)$, the flow curves are well described by the generalized Herschel Bulkley relation (3). The numerical values of the fitting parameters $\left(p, k, k, n, \sigma_{\mathrm{y}}\right)$ as well as the yield strain $\gamma_{\mathrm{y}}$ are reported in Table 2. One important result concerns the value of the exponent $p$ describing the low shear behaviour of the flow curves, which decreases when the concentration approaches $C_{\mathrm{J}}$. This observation is in agreement with recent MCT predictions for hard spheres entropic glasses. ${ }^{37}$ The high shear rate exponent $n$ also decreases as shown in Table 2.

In the jammed glass regime $\left(C>C_{\mathrm{J}}\right)$, the flow curves are characterized by a flat plateau at low shear rates $(p \cong 0)$ and a single power law variations at high shear rates. They are well fitted to the Herschel-Bulkley equation (2). The values of the yield stress $\sigma_{\mathrm{y}}$, yield strain $\gamma_{\mathrm{y}}\left(\gamma_{\mathrm{y}}=\sigma_{\mathrm{y}} / G_{0}\right)$ consistency $k$, and shear thinning exponent $\mathrm{n}$ are reported in Table 2 . We observe that the exponent $n$ is constant within the experimental uncertainties up to $C=30 \mathrm{mg} / \mathrm{g}(n=0.50 \pm 0.03)$ and decreases at higher concentrations. Secondly the ratio $\alpha=k / G_{0}$ is constant ( $\alpha=0.020 \pm 0.001$ ), indicating that the consistency parameter is dominated by elasticity.

These results can be interpreted using a micromechanical model which describes the flow properties of jammed particle

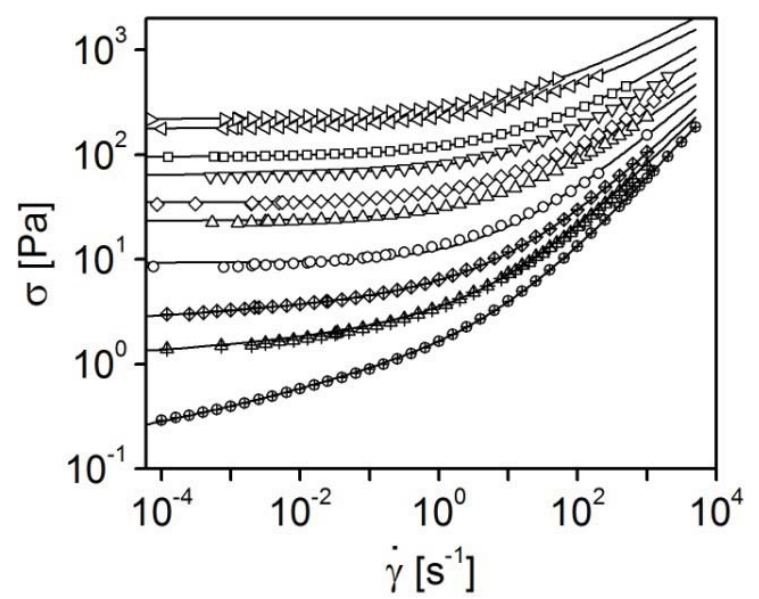

Fig. 7 Flow curves of entropic $[C=12(\oplus), 13(\oplus)$, and $14(\oplus) \mathrm{mg} / \mathrm{g}]$ and jammed glasses $[C=16(\bigcirc), 18(\triangle), 20(\diamond), 24(\nabla), 30(\square), 40(\triangleleft), 50(\triangleright) \mathrm{mg} / \mathrm{g}]$. Lines are fits of the data to Eq. $2\left(C_{G}<C<C_{J}\right)$ and Eq. $3\left(C>C_{J}\right)$.

\begin{tabular}{cccccccc}
\hline $\begin{array}{c}C \\
(\mathrm{mg} / \mathrm{g})\end{array}$ & $p$ & $\begin{array}{c}k^{\prime} \\
\left(\mathrm{Pa} . \mathrm{s}^{\mathrm{p}}\right)\end{array}$ & $n$ & $\begin{array}{c}k \\
\left(\mathrm{~Pa} . \mathrm{s}^{\mathrm{n}}\right)\end{array}$ & $\begin{array}{c}\alpha=k / G_{0} \\
\left(\mathrm{~s}^{\mathrm{n}}\right)\end{array}$ & $\begin{array}{c}\sigma_{y} \\
(\mathrm{~Pa})\end{array}$ & $\begin{array}{c}\gamma_{y} \\
(-)\end{array}$ \\
\hline 12 & 0.20 & 1.22 & 0.73 & 0.36 & 0.033 & 0.1 & 0.008 \\
13 & 0.17 & 1.77 & 0.66 & 0.75 & 0.023 & 1.0 & 0.031 \\
14 & 0.15 & 3.02 & 0.63 & 1.3 & 0.020 & 2.2 & 0.035 \\
16 & - & - & 0.55 & 3.2 & 0.021 & 9.3 & 0.061 \\
18 & - & - & 0.49 & 7.1 & 0.020 & 23.3 & 0.064 \\
20 & - & - & 0.48 & 9.25 & 0.019 & 35.0 & 0.069 \\
22 & - & - & 0.47 & 11.1 & 0.019 & 40.2 & 0.066 \\
24 & - & - & 0.46 & 14.8 & 0.019 & 64.2 & 0.078 \\
26 & - & - & 0.46 & 17.5 & 0.019 & 70.7 & 0.079 \\
30 & - & - & 0.43 & 24.9 & 0.021 & 95.2 & 0.082 \\
40 & - & - & 0.39 & 50.4 & 0.025 & 176.7 & 0.090 \\
50 & - & - & 0.39 & 68.7 & 0.035 & 218 & 0.095
\end{tabular}

Table 2 Values of experimental parameters determined from non linear rheology experiments of concentrated microgel suspensions in the entropic and jammed glass states. All the parameters result from fits of the flow curves to Eq. (3) and (4) except for the yield strain $\gamma_{y}$ which is defined as $\gamma_{y}=\sigma_{y} / G_{0}$. The values of $G_{0}$ used to compute $\alpha=k / G_{0}$ are given in Table 1

glasses. ${ }^{16}$ A glass is modelled as a dispersion of non-Brownian, elastic spheres with contact modulus $E^{*}$ which are dispersed at a volume fraction above random close-packing $(\phi>0.64)$ in a solvent of viscosity $\eta_{\mathrm{S}}$. Each particle has contacting facets with several neighbours. It is subjected to a net repulsive force and a net drag force which are the sum of the elastic and elastohydrodynamic forces at its contacting facets, respectively. The model predicts that the flow curves obey a constitutive equation of the Herschel-Bulkley form: $\sigma=\sigma_{\mathrm{y}}+k \dot{\gamma}^{1 / 2}$ with $\alpha=k / G_{0}=K\left(\eta_{\mathrm{S}} / E^{*}\right)^{1 / 2}$ where $K=80 \pm 3 . E^{*}$ is the particle contact modulus which depends on the Young modulus $E$ and the Poisson ratio $v: E^{*}=E / 2\left(1-v^{2}\right)$. This prediction matches well with the experimental results. One useful application is the determination of the particle contact modulus $E^{*}\left(=\eta_{\mathrm{S}} K^{2} / \alpha^{2}\right)$, which is unknown a priori and cannot be readily measured for submicron microgels: $E^{*}=16 \pm 1 \mathrm{kPa}$. The model also predicts a universal constitutive law: $\sigma / \sigma_{y}=1+\left(\eta_{s} \dot{\gamma} / \gamma_{y}^{2} E^{*}\right)^{1 / 2}$. To check the validity of our analysis, we replot the flow curves



Fig. 8 Master curve obtained by plotting the flow curves measured in the jammed regime (Fig. 7) in the set of coordinates derived from the elastohydrodynamic model $[C=16(\bigcirc), 18(\triangle), 20(\diamond), 22(\square), 24(\nabla), 26(+), 30(\square), 40(\triangleleft), 50(\triangleright) \mathrm{mg} / \mathrm{g}]$. The solid line is the theoretical flow curve expected from the model. 
shown in Fig. 7 using the non-dimensional stress $\sigma / \sigma_{\mathrm{y}}$ and rate $\eta_{s} \dot{\gamma} / \gamma_{y}^{2} E^{*}$. Fig. 8 shows that the flow curves for $C>16 \mathrm{mg} / \mathrm{g}$ collapse on a master curve which agrees with the expected constitute law. The data for the concentration $C=16 \mathrm{mg} / \mathrm{g}$, which is the closest to the jamming transition, deviates from the prediction. The data for the highest concentrations $C=40$ and $50 \mathrm{mg} / \mathrm{g}$ also collapse although the high shear rate exponent is different from the expected value 0.50 and the coefficient $\alpha$ is no longer constant suggesting again a change of behaviour.

Let us now discuss the yielding properties. Fig. 9 shows the variations of the yield strain determined by the strain sweep method in LAOS and the steady shear method. In the jammed regime, both data sets agree within the experimental accuracy. This is not the case in the glassy regime where the yield strain measured by LAOS clearly overestimates the value determined from the flow curve. This result is reminiscent of previous observations for star polymer solutions where yielding occurs in a range of shear rates although terminal regime is reached at low shear rates. ${ }^{32}$ The discrepancy comes from the fact that LAOS measurements probe the behaviour at a particular finite frequency whereas the yield strain measured by steady flow is obtained in the low frequency limit.

\subsection{Determination of the actual volume fraction of suspensions}

The rheological properties of soft particle dispersions are usually expressed in terms of the effective volume fraction computed from the single particle hydrodynamic size. ${ }^{42}$ Since microgels are able to shrink, the actual volume fraction can be very different from the effective volume fraction based on the low concentration limit. ${ }^{25,26,60}$ So far, very few studies have tried to determine the actual volume fraction of soft dispersions. Here we capitalize on the results presented in the previous sections to quantify the importance of deswelling and express a semi quantitative relation relating the experimental control parameter $C$ to the actual volume fraction $\phi$.

\section{Suspensions in the limit $C \rightarrow 0$}

For very dilute suspensions, a linear relation between $C$ and the volume fraction $\phi$ is found by mapping the viscosity variations

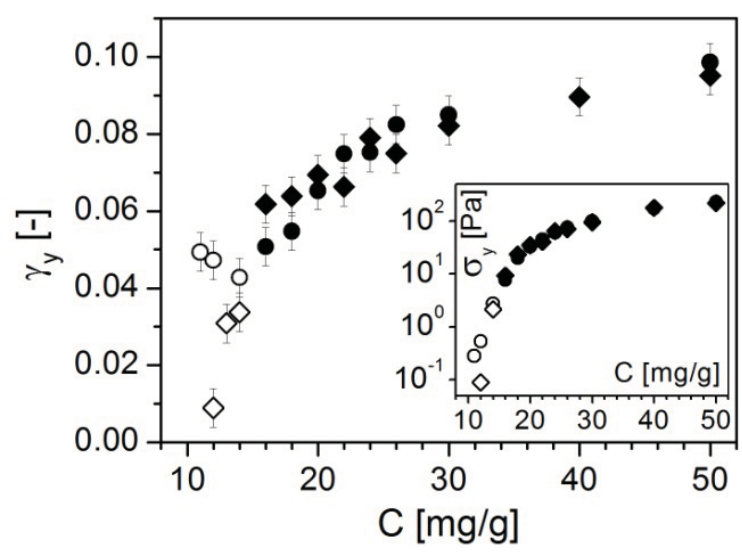

Fig. 9 Variations of the yield strain and yield stress (inset) versus the polymer concentration. Open symbols are relative to thermal glass state; full symbols refer to the jammed glass state. $\bigcirc, \bullet$ : data obtained from LAOS (Fig.4); $\diamond, \diamond$ : data obtained from flow curves (Fig. 7). described as a second-order polynomial in Fig. 6 to the Einstein-Batchelor equation for Brownian hard spheres (Eq. A1): $\phi_{0}=k C$ with $k=(0.11 \pm 0.01) \mathrm{g} / \mathrm{mg}$. This relationship is represented in Fig. 10; it predicts that microgel dispersions should be solid at $C=7 \mathrm{mg} / \mathrm{g}$, which is not the case in our experiments due to deswelling.

\section{Dilute suspensions}

Swelling at finite concentrations is regulated by the distribution of ions inside and outside the particles. In crosslinked microgels most of the counterions associated with the ionized units are trapped inside the microgel by the electric field created by the fixed charges borne by the polymer; only a small fraction $\Gamma(\cong 0.15)$ of counter ions are free to move in the suspending solution. ${ }^{25}$ When the volume fraction of the particles increases, the free counterions are confined in a smaller volume, their concentration increases, and the net osmotic pressure decreases causing the deswelling of the microgels.

This problem has been addressed independently by two different groups. ${ }^{25,26}$ The following expression was shown to relate the actual volume fraction to the volume fraction $\phi_{0}$ estimated in the limit $C \rightarrow 0$ :

$$
\left(\frac{\phi}{\phi_{0}}\right)^{2 / 3}=1-\frac{\Gamma}{1-\Gamma} \frac{\phi}{1-\phi}
$$

This relation is valid for $\phi<1-\Gamma$. At higher volume fractions the counter ions concentration inside and outside the microgels are equal so that osmotic deswelling becomes negligible. The validity of prediction (5) has been successfully tested. ${ }^{25,26}$ In Fig. 10, we have plotted the volume fraction obtained by solving Eq. (5) with $\Gamma=0.15$. We find that the relation between the concentration and the actual volume fraction is highly non linear. Deswelling in the dilute suspension regime is a significant effect. The linear relation $\phi_{0}(C)$ is only valid at very low volume fractions $(\phi<0.2)$. At $C=10 \mathrm{mg} / \mathrm{g}$, i.e. close to the glass transition, microgels deswell by $40 \%$ in volume and their radius is reduced to about $260 \mathrm{~nm}$.

The model also provides expressions for the concentration of counterions inside and outside the microgels: $C_{\text {in }}=f(1-\Gamma) c$ and $C_{\text {out }}=f \Gamma c \phi /(1-\phi)(f$ : ionization degree; $c$ : methacrylic acid concentration inside the microgels). From these relations we can estimate the effective charge borne by the microgels, $\sigma=$ $0.01 \mathrm{e} / \mathrm{nm}^{2}$ and the Debye lengths inside and outside the microgels, $\Lambda_{\text {in }}$ and $\Lambda_{\text {out }}{ }^{25,43}$ The effective charge is very low and $\Lambda_{\text {out }}$ becomes rapidly much smaller that the microgel radius when $\phi$ increases $\left(\Lambda_{\text {out }} \cong 10 \mathrm{~nm}\right.$ for $\left.\phi=0.1\right)$. In conclusion, the free counterions suffice to screen the repulsive electrostatic interactions due to the weak charge borne by the microgels even in the absence of any residual salt. This suggests that the rheological behaviour of microgel suspensions is dominated by excluded volume interactions like in hard sphere suspensions.

To test this conclusion, we compare the experimental data presented in Fig. 6 with the theoretical values expected for hard sphere suspensions (Appendix). For the low shear viscosity we use Eqs A2 and A3 with $\phi_{\mathrm{G}}=0.615$ being the value of the volume fraction predicted by Eq. 5 for $C_{\mathrm{G}}=11 \mathrm{mg} / \mathrm{g}$. Given the experimental uncertainties which affect the determination of the 
volume fractions, we can consider that the experimental data and the theoretical predictions are in reasonable agreement.

\section{Entropic glasses}

In the entropic glass state, the osmotic deswelling model remains valid and can also be used to quantify $\phi(C)$ (Fig. $10)$. The glass transition is found to occur at $\phi_{\mathrm{G}}=0.615$, a value slightly larger than the established value $\phi_{\mathrm{g}}=0.58$ for conventional hard spheres. Different reasons can explain the discrepancy: the polydispersity of our microgel suspensions but also the uncertainties which are inherent to the derivation of the $\phi(C)$ relation. The model predicts that deswelling becomes less and less important as the polymer concentration increases. This is because the concentrations of counterions inside and outside the microgels tend to become equal.

\section{Jammed glasses}

In the jammed glass state, Eq. 5 no longer applies. We relate $\phi$ and $C$ by comparing the experimental variations of $G_{0} / E^{*}$ with the concentration to the theoretical form: ${ }^{24} G_{0} / E^{*}=0.19\left(\phi-\phi_{\mathrm{J}}\right)$. We use the values of $G_{0}$ given in Table 1 and $E^{*}=16 \mathrm{kPa}$. We observe that the volume fraction increases linearly with the concentration, which signals that osmotic deswelling is negligible in this regime, as expected from the model. The counterion distribution is essentially uniform over the suspension. At the jamming point, the predicted volume fraction $\phi_{\mathrm{J}}=0.66$ falls slightly above the expected value $\phi_{\mathrm{J}}=0.64$ but again we are limited by experimental uncertainties.

\section{Dense glasses}

The volume fraction approaches its maximum value 1 for $C \cong 30 \mathrm{mg} / \mathrm{g}$. At these concentrations, the microgels are packed into dense suspensions without significant free volume left between them. In dense glasses, the actual volume fraction remains close to 1 and microgels cannot absorb enough water to reach swelling equilibrium. We call steric deswelling this new mechanism according to which the particles accommodate the steric constraints by reducing their size. The linear and non linear data reported in the previous sections indicate systematic deviations from the behaviour of jammed glasses (see for instance Figs 3 and 4 as well as Table 2). A more systematic study would be useful to determine whether these deviations come from the changes in the individual microgel properties (size, Young modulus, osmotic compression) or more fundamentally from a breakdown of the micromechanical model.

\section{Concluding remarks}

The colloidal microgels studied in this manuscript have a softness parameter $\varepsilon=E^{*} R^{3} / k T \cong 10^{5}$, which is intermediate between parameters of emulsions and ultrasoft particles like star polymers. They exhibit a rich state diagram with well separated glass and jamming transitions, and four different states: colloidal suspension, entropic glass, jammed glass, and dense glass. The crossover we observe between entropic elasticity and jamming elasticity is reminiscent of the behaviour



Fig. 10 Relations between the polymer concentration $C$ and the actual volume fraction $\phi$. Open symbols $(O)$ and continuous line: data calculates from the osmotic deswelling model. Full symbols $(\mathbf{O})$ : data obtained by mapping the flow curves to predictions for jammed athermal elastic spheres. The dashed line represents the relation determined in the absence of deswelling. Star symbols (*) represent the location of the glass and jamming transitions expected for hard spheres at $\phi_{\mathrm{G}}=0.58$ and $\phi_{\mathrm{J}}=0.64$ respectively.

of concentrated emulsions. This result somewhat contradicts previous observations that dynamical arrest in microgels is controlled by the glass transition and that they should not be well suited to study the jamming transition. The state diagram is strongly affected by the particle softness and in particular the capacity of microgels to deswell. Although the determination of the volume fraction of a suspension is a difficult task, we have been able to account for particle deswelling and get reasonable estimates of the actual volume fraction. The glass and jamming transitions seem to occur slightly above the values expected for hard spheres. Despite the experimental uncertainties inherent to our method, we can argue that the effect of softness on the location of the glass and jamming transition is negligible in our system. More accurate determinations of the volume fraction would be useful to better disentangle deswelling effects from rheology.

In the dilute suspension regime, where osmotic deswelling plays an important role, microgel suspensions have a purely viscous behaviour. The variations of the low and high shear viscosities with the concentration appear to be softer than for hard spheres. However, once expressed in terms of the actual volume fraction, the data closely match the variations for hard sphere suspensions. In the jammed glass state, osmotic deswelling becomes negligible. The flow curves can be rationalized in terms of a micromechanical model incorporating elastic and elastohydrodynamic forces. This highlights the importance of elastic contacts between interlocked neighbouring particles. In the dense regime at high concentration, the actual volume fraction remains close to its maximum value 1 . The microgels accommodate the constraints by sterically deswelling. Systematic deviations from the predictions are found, which raises the question of the validity of the micromechanical model for dense suspensions. Additional experiments coupling particle scale rheology and microstructure analysis would be useful to go further. 
These results highlight two important manifestations of softness, i.e. elastic deformations and volume changes, which act independently. In that respect, we think that our findings represent generic behaviours which should be found in many other systems once the proper protocol to deal with such soft systems is recognized and implemented. More complex situations where interdigitation between particles takes place remain a challenging issue. This calls for future investigations of hybrid architectures such as star polymers and well controlled hairy microgels.

\section{Appendix: models for the viscosity of hard sphere suspensions}

The modelling of the viscosity of hard sphere suspensions has stimulated a lot of work and reasonable predictions are now available. In the very dilute regime when $\phi \rightarrow 0$, the low shear relative viscosity of Brownian hard sphere suspensions is well described by the following equation first derived by Batchelor: ${ }^{61}$

$$
\frac{\eta_{0}}{\eta_{\mathrm{s}}}=1+2.5 \phi+6.2 \phi^{2}
$$

At higher concentration an expression based on MCT is known to correctly capture the increase of the relative low shear viscosity in the dilute colloidal suspension regime and the divergence at the glass transition $\phi_{\mathrm{g}}=0.58 \mathrm{:}^{62}$

$$
\frac{\eta_{0}-\eta_{\infty}^{\prime}}{\eta_{S}}=\frac{0.9 \phi^{2}}{\left(1-\phi / \phi_{g}\right)^{2.46}}
$$

The high frequency relative viscosity is modelled by the validated semi-empirical correlation: ${ }^{63}$

$$
\frac{\eta_{\infty}^{\prime}}{\eta_{\mathrm{S}}}=\frac{1+1.5 \phi\left(1+\phi-0.189 \phi^{2}\right)}{1-\phi\left(1+\phi-0.189 \phi^{2}\right)}
$$

Finally in the literature, the high shear relative viscosity is well represented by the correlation: ${ }^{64}$

$$
\frac{\eta_{\infty}}{\eta_{\mathrm{S}}}=\left(1-\frac{\phi}{0.71}\right)^{-2}
$$

\section{Acknowledgments}

We are indebted to Roger T. Bonnecaze and Lavanya Mohan for enlightening discussions on the rheology of microgels suspensions. We acknowledge Dimitris Vlassopoulos for stimulating discussions on the role of softness in soft particle rheology and for a critical reading of the manuscript. We thank Maddalena Mattiello for help in experiments.

\section{References}

1 P. N. Pusey and W. van Megen, Nature, 1986, 320, 1986.

2 P. N. Pusey, J. Phys.: Condens. Matter, 2008, 20, 494202.

3 P. N. Pusey, in Liquids, Freezing and Glass Transition, ed. J. P. Hansen, D. Levesque and J. Zinn-Justin, North-Holland, Amsterdam, 1991.

4 G. Petekidis, D. Vlassopoulos and P. N. Pusey, J. Phys.: Condens. Matter, 2004, 16, S3955.

5 L. Marshall and C. F. Zukoski, J. Phys. Chem., 1990, 94, 1164.

6 T. G. Mason and D. A. Weitz, Phys. Rev. Lett., 1995, 75, 2770 .

7 G. Petekidis, A. Moussaïd and P. N. Pusey, Phys. Rev. E, 2002, 66, 051402 .

8 G. Brambilla, D. El Masri, M. Pierno, L. Berthier and L. Cipelletti, Phys. Rev. Lett., 2009, 102, 085703.

9 G. Petekidis, D. Vlassopoulos and P. N. Pusey, Faraday Discuss., 2003, 123, 287.

10 N. Koumakis, M. Laurati, S. U. Egelhaaf, J. F. Brady and G. Petekidis, Phys. Rev. Lett., 2012, 108, 098303.

11 M. Fuchs and M. E. Cates, Phys. Rev. Lett., 2002, 89, 248304

12 M. Fuchs and M. E. Cates, J. Rheol., 2009, 53, 957.

13 E. J. Saltzman, G. Yatsenko and K. S. Schweizer, J. Phys.: Matter, 2008, 20, 244129.

14 J. M. Brader, T. Voigtmann, M. Fuchs, R. G. Larson and M. E. Cates, Proc. Natl. Acad. Sci. U.S.A., 2009, 106, 15186.

15 R. T. Bonnecaze and M. Cloitre, Adv. Polym. Sci., 2010, 236, 117.

16 J. Seth, L. Mohan, C. Locatelli-Champagne, M. Cloitre and R. T. Bonnecaze, Nat. Mat., 2011, 10, 838.

17 A. Ikeda, L. Berthier and P. Sollich, Phys. Rev. Lett., 2012, 109, 018301.

18 D. Vlassopoulos, M. Cloitre, Curr. Opin. Colloid Interface Sci., 2014, 19, 561.

19 M. Cloitre, R. Borrega, F. Monti, and L. Leibler, Phys. Rev. Lett., 2003, 90, 068303.

20 M. van Hecke, J. Phys.: Condens. Matter, 2010, 22, 033101.

21 B. P. Tighe, E. Woldhuis, J. J. C. Remmers, W. van Saarloos and M. van Hecke, Phys. Rev. Lett., 2010, 105, 088303.

22 C. S. O'Hern, L. E. Silbert, A. J. Liu and S. R. Nagel, Phys. Rev. E, 2003, 68, 011306

23 C. N. Likos, H. Löwen, M. Watzlawek, B. Abbas, O. Jucknischke, J. Allgaier and D. Richter, Phys. Rev. Lett., 2003, 80, 4450 .

24 J. R. Seth, M. Cloitre and R. Bonnecaze, J. Rheol., 2006, 50, 353.

25 R. Borrega, M. Cloitre, I. Betremieux, B. Ernst and L. Leibler, Europhys. Lett., 1999, 47, 729.

26 G. Romeo, L. Imperiali, J.-W. Kim, A. Fernández-Nieves and D. A. Weitz, J. Chem. Phys., 2012, 136, 124905.

27 D. Vlassopoulos and G. Fytas, Adv. Polym. Sci., 2010, 236, 1.

28 H. Gang, A. H. Krall, H. Z. Cummins and D. A. Weitz, Phys. Rev. E, 1999, 59, 715.

29 T. G. Mason, J. Bibette and D. A. Weitz, Phys. Rev. Lett., 1995, 75, 2051.

30 F. Scheffold, F. Cardinaux and T. G. Mason, J. Phys.: Condens. Matter, 2013, 25, 502101.

31 T. G. Mason and F. Scheffold, Soft Matter, 2014, 10, 7109.

32 B. M. Erwin, M. Cloitre, M. Gauthier and D. Vlassopoulos, Soft Matter, 2010, 6, 2825

33 A. Ikeda, L. Berthier and P. Sollich, Soft Matter, 2013, 9, 7669.

34 Microgel Suspensions: Fundamentals and Applications, ed. A. Fernandez-Nieves, H. Wyss, J. Mattsson and D. A. Weitz, Wiley, 2011.

35 M. Fuchs and M. Ballauff, J. Chem. Phys., 2005, 122, 94707. 
36 J. J. Crassous, M. Siebenbürger, M. Ballauff, M. Drechsler, O. Henrich and M. Fuchs, J. Chem. Phys., 2006, 125, 204906.

37 M. Siebenbürger, M. Fuchs, H. Winter and M. Ballauff, $J$. Rheol., 2009, 53, 707.

38 V. Carrier and G. Petekidis, J. Rheol., 2009, 53, 245.

39 D. Paloli, P. S. Mohanty, J. J. Crassous, E. Zaccarelli and P. Schurtenberger, Soft Matter, 2013, 9, 3000-3004.

40 K. N. Nordstrom, P. E. Arratia, E. Verneuil, A. Basu, Z. Zhang, A. G. Yodh, J. P. Gollub and D. J. Durian, Phys. Rev. Lett., 2010, 105, 175701.

41 A. Basu, Y. Xu, T. Still, P. E. Arratia, Z. Zhang, K. N. Nordstrom, J. M. Rieser, J. P. Gollub, D. J. Durian and A. G. Yodh, Soft Matter, 2014, 10, 3027-3035.

42 J. Mattsson, H. M. Wyss, A. Fernandez-Nieves, K. Miyazaki, Z. Hu, D. R. Reichman and D. A. Weitz, Nature, 2009, 462, 83.

43 B. Sierra-Martin and A. Fernandez-Nieves, Soft Matter, 2012, 8, 4141

44 M. Pelaez-Fernandez, A. Souslov, L. A. Lyon, P. M. Goldbart and A. Fernandez-Nieves, Phys. Rev. Lett. 2015, 114, 098303.

45 M. Cloitre, R. Borrega, F. Monti and L. Leibler, C. $R$. Physique, 2003, 4, 221.

46 R. Acciaro, T. Gilanyi and I. Varga, Langmuir, 2011, 27, 7917.

47 T. Still, K. Chen, A. M. Alsayed, K. B. Aptowicz and A. G. Yodh, J. Colloid Int. Sci., 2013, 405, 96.

48 F. Scheffold, P. Díaz-Leyva, M. Reufer, N. Ben Braham, I. Lynch and J. L. Harden, Phys. Rev. Lett., 2010, 104, 128304; G. Romeo and M. P. Ciamarra, Soft Matter, 2013, 9, 5401.
49 M. Cloitre, R. Borrega and L. Leibler, Phys. Rev. Lett., 2000, $85,4819$.

50 R. B. Bird, R. C. Armstrong and O. Hassager, Dynamics of Polymeric Liquids, John Wiley \& Sons, New York, 1987.

51 K. Schâtzel, Appl. Optics, 1993, 32, 3880.

52 S. W. Provencher and P. Stepanek, Part. Part. Syst. Charact. 1996, 13, 291.

53 G. Petekidis, J. Gapinski, P. Seymour, J.S. van Duijneveldt and G. Fytas, Phys. Rev. E, 2004, 69, 042401.

54 P. N. Segrè and P. N. Pusey, Phys. Rev. Lett., 1996, 77, 771.

55 R. Sigel, S. Pispas, D. Vlassopoulos, N. Hadjichristidis and G. Fytas, Phys. Rev. Lett. 1999, 83, 4667

56 P. Holmqvist, P.S. Mohanty, G. Nägele, P. Schurtenberger and M. Heinen, Phys. Rev. Lett., 2012, 109, 048302.

57 L. Mohan, C. Pellet, M. Cloitre and R. Bonnecaze, J. Rheol., 2013, 57, 1023

58 T. G. Mason, J. Bibette and D. A. Weitz, J. Coll. Interf. Sci., 1996, 179, 439.

59 N. Merlet-Lacroix, E. Di Cola and M. Cloitre, Soft Matter, $2010,6,984$.

60 U. Gasser, J. S. Hyatt, J.-J. Lietor-Santos, E. S. Herman, L. A. Lyon and A. Fernandez-Nieves, J. Chem. Phys., 2014, 141, 034901.

61 G. K. Batchelor, J. Fluid Mech., 1977, 83, 97.

62 W. B. Russel, N. J. Wagner and J. Mewis, J. Rheol., 2013, 57, 1555.

63 R. A. Lionberger and W.B. Russel, Adv. Chem. Phys., 2000, 111, 399.

64 J. C. van der Werff and C. G. de Kruif, J. Rheol., 1989, 33, 421. 